\title{
THE UTILIZATION OF RAPID SEROLOGICAL TESTS IN COVID-19 DIAGNOSTICS - A HIGH RISK OF FALSE-NEGATIVE RESULTS IN OUTPATIENT CARE, WITH PARTICULAR EMPHASIS ON DENTAL TREATMENT
}

\author{
Monika Tysiąc-Miśta ${ }^{1}$, Sylwia Bulanda ${ }^{2}$ \\ Medical University of Silesia in Katowice, Katowice, Poland \\ Faculty of Medical Sciences in Zabrze \\ ${ }^{1}$ Department of Dental Materials, Chair of Prosthetics and Dental Materials \\ ${ }^{2}$ Department of Chemistry
}

\begin{abstract}
In order to mitigate the spread of COVID-19, in the early stages of the pandemic outbreak, postponing elective procedures was recommended all around the world. Outpatient care and dental care were limited to telephone advice and emergency services. Dental staff is particularly vulnerable to SARS-CoV-2 contraction, because of the inevitable contact with patients' body fluids during aerosol-generating procedures. The implementation of diagnostic tests among ambulatory patients could improve the occupational safety among outpatient care personnel. The aim of this review was to introduce information regarding COVID-19 diagnostics with a particular focus on the methods which can be utilized in an outpatient and dental care setting. An online PubMed database review of articles on COVID-19 diagnostics, published on February 12-May 15, 2020, was conducted. Reverse transcription polymerase chain reaction is the gold standard in COVID-19 diagnostics, which determines if a person has an active infection. Unfortunately, its utilization in outpatient care is limited. Serological enzyme-linked immunosorbent assays identify people who were infected, including those who have had an asymptomatic infection, but they do not give sufficient information about the acute infection. Rapid serological assays developed to facilitate testing outside of laboratories, especially in dental offices, are not recommended by the World Health Organization to be used outside research settings, and they should not constitute the basis for clinical decision-making because of frequent false-negative results which may consequently contribute to personnel infections. Out of all available COVID-19 diagnostic methods, rapid serological assays seemed to be a method of choice in outpatient medical care. Unfortunately, their results turned out to be unreliable. The best methods to ensure the occupational safety of medical staff and to avoid cross-infections in outpatient care facilities include a thorough epidemiological interview, temperature measurement to rule out patients with an active infection, and the implementation of strict infection control procedures. Med Pr. 2021;72(2):155-62
\end{abstract}

Key words: dentistry, serology, rapid test, laboratory diagnostics, COVID-19, SARS-CoV-2

Corresponding author: Monika Tysiąc-Miśta, Medical University of Silesia in Katowice, Faculty of Medical Sciences in Zabrze, Department of Dental Materials, Chair of Prosthetics and Dental Materials, Plac Akademicki 17, 41-902 Bytom, Poland, e-mail: monikatysiac@wp.pl

Received: June 8, 2020, accepted: September 9, 2020

\section{INTRODUCTION}

Since December 8, 2019, a series of pneumonia cases of an unknown origin has emerged in Wuhan, Hubei Province, China. In the beginning of this disease, severe acute respiratory infection symptoms occurred, with some patients rapidly developing acute respiratory distress syndrome, acute respiratory failure, and other serious complications [1]. On January 7, 2020, a novel coronavirus was identified by the Chinese Center for Disease Control and Prevention (CCDCP) from a patient's throat swab [2]. On February 11, the International Committee on Taxonomy of Viruses officially designated the SARS-CoV-2 virus [3]. As the COVID-19 pandemic accelerated, on March 11, 2020, the World Health Organization (WHO) declared COVID-19 a pandemic [4].

Effective testing gives a chance of sufficient contact tracing and containing the spread of the disease from the very beginning. Unfortunately, due to scarce testing capacity, nearly all countries in the world decided to implement social distancing measures. On March 16, 2020, 
the American Dental Association (ADA) recommended postponing elective dental procedures [5]. The aim of this recommendation was to mitigate the spread of the coronavirus in the dental offices setting, as the utilization of prolonged aerosol-generating procedures constitutes a high risk of COVID-19 contraction [6]. Then, as governments and dental associations around the world lifted their mandates, dentists reopened their practices [7]. Just until August 15, 2020, 20995433 laboratory confirmed COVID-19 cases had been identified, and 760774 people had died [8].

In an ideal situation, when a patient is admitted to a dental office, he or she should show a laboratory test result, either molecular or serological. Molecular diagnostics is expensive and time-consuming, but an interesting alternative is provided by a rapid immunodiagnostic test for COVID-19. Multiple diagnostic test manufacturers have developed and begun selling rapid and easy-to-use devices to outpatient clinics in order to facilitate testing outside of laboratories [9]. The aim of this paper is to investigate the available COVID-19 diagnostic methods, which can be utilized in outpatient clinics, with special consideration of dental offices, to ensure the occupational safety of both medical staff and their patients.

\section{METHODS}

Using online databases, a systematic review of literature concerning COVID-19 diagnostics and its implications for dentistry was carried out. This review is based on scientific articles which were available in the PubMed database. Supplementary information was obtained from WHO and ADA, and from the Food and Drug Administration (FDA) websites. The key words utilized included: "SARS-Co-2," "coronavirus," "COVID-19," "diagnostic tests," "molecular tests," "laboratory diagnostics," "RT-PCR," "serology," "antibody tests," "ELISA," "antigen tests," "rapid tests," "point-of-care tests," "saliva," "blood," "serum," "swab tests," "dentistry," "dental office," and "dentists." The authors included scientific papers published on January 24-May 15, 2020, both in Polish and English languages. Only publications focusing on the specific scope of this review were eligible for inclusion.

\section{RESULTS}

\section{Characteristics of SARS-CoV-2}

Coronaviruses (CoVs) are a group of positive-sense, single-stranded RNA viruses [10]. They belong to subfamily Orthocoronavirinae, family Coronaviridae, suborder Cornidovirineae, and order Nidovirales [11]. The Orthocoronavirinae are further divided into 4 genera: $\alpha-\mathrm{CoV}, \beta-\mathrm{CoV}, \gamma-\mathrm{CoV}$, and $\delta-\mathrm{CoV}$ [12]. The $\alpha-\mathrm{CoV}$ and $\beta-\mathrm{CoV}$ genera mainly infect the respiratory, gastrointestinal, hepatic and central nervous systems of mammals [13]. Based on the sequence analysis of the viral genome, SARS-CoV, MERS-CoV and SARS-CoV-2 belong to $\beta$-CoV. The nucleotide sequence similarity between SARS-CoV-2 and SARS-CoV is 79\%, and between SARS-CoV-2 and MERS-CoV about 50\% [14]. The genome nucleotide sequence indicated that the natural host of SARS-CoV-2 is Rhinolophus affinis bat $[15,16]$. The genome of SARS-CoV-2 encodes 4 structural proteins (including Spike $[\mathrm{S}]$ and Nucleocapsid $[\mathrm{N}]$ ), 8 accessory proteins, and 15 non-structural proteins [17]. The $\mathrm{S}$ protein comprises the receptor binding domain which is responsible for binding to the angiotensin-converting enzyme 2 membrane receptor of the host cell [18]. The $\mathrm{N}$ protein is essential for the packaging and replication of the virus. The $S$ and $\mathrm{N}$ proteins demonstrate high antigenicity [19].

A recent study has indicated that the highest viral load is found immediately after the onset of symptoms, and in greater quantities in the nose than in the throat. Compared to the typical flu, COVID-19 is much more lethal with a mortality rate of about $2.92 \%$, whereas the annual flu has a mortality rate of just $0.05-0.1 \%$ [20]. The viral load found in asymptomatic COVID-19 subjects was similar to that of symptomatic ones, which suggests the potential transmission role of asymptomatic subjects [21]. The inter-human transmission routes of CoVs include droplets, direct contact and indirect contact through surfaces. The virus has also been isolated in the serum, blood, rectal swabs, saliva, urine and stool [14]. As the incubation period ranges 0-24 days, transmission can occur before any symptoms become apparent [22]. When a patient is suspected of the SARSCoV-2 infection, he or she is quarantined for 14 days. During that time, a molecular swab test is carried out. Unfortunately, if the result of the test is false-negative and the patient does not develop COVID-19 symptoms, he or she is unaware of being an asymptomatic carrier of the disease. This poses a great occupational threat to the medical personnel treating such a patient.

\section{Specimen collection}

According to WHO recommendations, specimens for molecular diagnostics can be collected from the upper and lower respiratory track. The U.S. Center for Disease 
Control and Prevention (CDC) recommends collecting the upper respiratory nasopharyngeal (NP) swab. The collection of an oropharyngeal (OP) specimen is a lower priority, and, if collected, it should be combined in the same tube as the NP swab [23]. The upper respiratory swab collection promotes bleeding, especially in patients with thrombocytopenia or those taking anticoagulant and antiplatelet agents. It can also determine gag and cough reflex, which, due to the proximity of the healthcare worker-patient contact, creates a high risk of virus transmission $[23,24]$. For the most sensitive detection of SARS-CoV-2, the collection and testing of both upper and lower respiratory samples (bronchoalveolar lavage fluid, endotracheal aspirates and expectorated sputum) is recommended [25]. However, according to Mizumoto et al. [26,27], only $28 \%$ of patients with the severe form of COVID-19 produce expectorant sputum. Lower respiratory tract samples are reserved for selected hospitalized or intensive care unit cases.

The accuracy of viral swab tests in clinical practice varies depending on the location and quality of sampling. In one study, reverse transcription polymerase chain reaction (RT-PCR) sensitivity in SARS-CoV-2 detection, in a group of 205 patients, was $93 \%$ for bronchoalveolar lavage, $72 \%$ for sputum, $63 \%$ for nasal swabs, and only $32 \%$ for throat swabs [28-30].

Sabino et al. [31] suggested that saliva might play a key role in SARS-CoV-2 human-to-human transition and non-invasive saliva-based diagnostics can be a convenient method for an early detection of the COVID-19 infection [31]. The virus can appear in the saliva via different routes: firstly, with expectorant secretion of the lower and upper respiratory tract [32]. Secondly, SARS-CoV-2 present in the blood can gain access to the oral cavity through gingival pockets that contain liquid with proteins, which are derived from the extracellular matrix and serum. Finally, SARS-CoV-2 can infect salivary glands. It should be emphasized that salivary epithelial cells are infected at the very beginning, suggesting that they may be a key source of the virus before general symptoms appear [33]. A recent test conducted by the Yale University School of Public Health found that saliva yielded greater detection sensitivity and consistency throughout the course of infection when compared with samples taken with nasopharyngeal swabs [34].

The FDA has granted the Emergency Use Authorization for a test that collects saliva instead of using the traditional nasal swab [35]. A great advantage of this procedure is the fact that patients can collect the specimens by themselves, not exposing the medical personnel to the danger of COVID-19 contraction. Some strains of SARS-CoV-2 have been detected in saliva up to 29 days after infection [31]. Serological assays require plasma, serum, blood, saliva, urine and fecal specimens. The material should be collected, stored and transported in accordance with valid recommendations $[36,37]$. Plasma or serum are the most frequently collected materials for serological tests. Some rapid tests also utilize a venous or capillary blood sample from the patient's finger, and material obtained from the respiratory tract $[30,38]$. Moreover, SARS-CoV-2 specific immunoglobulin A ( $\operatorname{Ig} \mathrm{A})$ production has been demonstrated in the saliva of intranasally immunized animals [39]. Accordingly, it is speculated that COVID-19 saliva-based diagnostics using specific antibodies against the virus may also be performed [31].

\section{Diagnostic tests}

Symptomatic COVID-19 patients are subjected to computer tomography (CT) scans and X-ray examinations of the lungs $[32,40]$. The British Society of Thoracic Imaging recommends CT imaging even in patients with a suspected coronavirus disease, with clinical symptoms and in poor health [41]. Apart from CT, lung ultrasound (US) is also recommended. Peng et al. [42] indicated 5 main US clinical findings, including the thickening of the irregular pleural line. Most COVID-19 patients also demonstrate changes in biochemical and morphological tests. Inflammatory parameters such as C-reactive protein, ferritin, procalcitonin and lactate dehydrogenase are increased. In addition, elevated D-dimer levels, fibrinogen and extended prothrombin time are often observed [43]. Among the tests for the COVID-19 infection, molecular tests, antigen tests, and serological tests, including rapid tests, can be distinguished [9]. This paper covers the available diagnostic methods which can be utilized in outpatient care, especially in dentistry.

\section{Molecular diagnostic tests}

As soon as a complete genome of SARS-CoV-2 was sequenced by CCDCP, the data was immediately published in international database banks [31]. This gave the possibility to establish specific primers and standard operating procedures for nucleic acid tests (NATs). The current NATs for RNA viruses mainly include RT-PCR, alternative isothermal amplification methods, and clustered regularly interspaced short palindromic repeats (CRISPR-Cas13a) based on the Specific 
High Sensitivity Enzymatic Reporter UnLOCKing (SHERLOCK) system [44]. Due to its simplicity, easy methodology and an extensively validated standard operating procedure, RT-PCR is now the preferred and most widely used method for NATs [36]. It is also considered the diagnostic gold standard, essential to confirm a SARS-CoV-2 infection [37]. Numerous tests have confirmed high sensitivity and specificity of this method [45]. It is estimated that the sensitivity of the test ranges $71-98 \%$ [29]. Due to the fact that RT-PCR is a quantitative method [46], a week after the first clinical symptoms appear, the sensitivity of this method gradually decreases due to the reduction in the number of viral particles in the epithelium of the respiratory tract, and the risk of false-negative results increases [36]. Reverse transcription polymerase chain reaction demonstrates the highest sensitivity between the seventh and 14th day after contact with SARS-CoV-2 [45].

The CDC proposed a SARS-CoV-2 identification algorithm based on a set of oligonucleotides, including nucleocapsid protein specific genes $(\mathrm{N} 1, \mathrm{~N} 2, \mathrm{~N} 3)$ and the RP gene. Current molecular diagnostics is based on ready-to-use real-time RT-PCR kits. All tests with questionable results, negative test results, when at the same time the patient manifests COVID-19 symptoms or has had contact with people who tested positive, should be repeated using a newly collected sample [45]. Typically, $>24 \mathrm{~h}$ are needed to perform the test [47]. There is an obligation to send samples to reference laboratories, which significantly extends the time one has to wait for the result [46]. Diagnostic protocols require equipment, reagents and trained medical personnel. Due to its shortages, the results may be obtained even after several days [26].

The collection of high-quality swabs requires qualified healthcare professionals, because the virus titer in specimens obtained from the upper respiratory tract swabs is much lower than in samples collected from the lower respiratory tract. In addition, the viral RNA titers vary at different stages of the infection [45], and the possibility of their detection in the upper respiratory tract decreases with time. [30] In a study by Zhang et al. [48], a positive test result was the highest at week 1 (100\%), followed by $89.3 \%, 66.1 \%, 32.1 \%, 5.4 \%$ and $0 \%$ at week 2 , week 3 , week 4 , week 5 and week 6 , respectively. Another disadvantage of the test is its relatively high cost. Other molecular tests, which are still under development and validation, and have not been approved yet, include reverse transcription loop-mediated isothermal amplification. This is 1-step nucleic acid amplification method which is fast but also difficult to perform, which tends to discourage researchers [49].

In general, the global shortage of diagnostic tests and swabs used for collecting respiratory samples, the frequency of false-negative results, and the inability of these tests to be performed in a quick and bulk way, which is especially required during the clinical admittance of patients, have highlighted the necessity for the development of additional testing methods [50].

\section{Antigen testing}

The FDA has authorized the emergency use of SARS$\mathrm{CoV}-2$ antigen testing. It is a type of a diagnostic test which detects the presence of viral proteins (antigens) in a sample obtained from the nasal swab specimen. The antigens are detected only at the initial stage of the disease when the virus is actively replicating; therefore, such tests are best used to identify acute or early infections. The result is obtained faster than in RT-PCR. Although the test is characterized by high specify, it also has low sensitivity and, therefore, gives a large percentage of false-negative results. For this reason, its result should be confirmed by RT-PCR [51,52]. The use of rapid antigen testing for SARS-CoV-2 infections under non-testing conditions is not recommended by WHO. Such tests should not be taken into account when making decisions by a doctor [9].

\section{Serological antibody diagnostics}

Serological tests are another laboratory method for the confirmation of COVID-19 diagnosis. They were developed in response to the shortages of laboratory-based molecular testing capacity and duration of test results $[29,53]$. They mainly include an enzyme-linked immunosorbent assay (ELISA), a chemiluminescence assay, an immunofluorescence assay, a western blot, a protein microarray, and a neutralization assay. Of these methods, ELISA is suitable for first-line screening due to its large throughput, short processing time, and a simple operating procedure, while the neutralization assay is used as the gold standard for confirmation in many laboratories [44].

Laboratory tests for antibodies utilize plasma or blood serum. However, some rapid tests use capillary blood or material collected from the patient's respiratory tract [26]. The tests screen for the presence of antibodies produced during the immune response against SARS-CoV-2 N protein [54]. They appear in the blood 1-3 weeks after the onset of symptoms and may persist for a lifetime. These tests can identify people who were 
infected and have already recovered from COVID-19, including those who have had an asymptomatic infection, in order to confirm it. They are not used to recognize an acute COVID-19 infection. Instead, they can be used to determine the percentage of a population that have contracted the disease and are, therefore, presumably immune. High IgG antibody titers indicate the acquisition of resistance to re-infection although the duration and effectiveness of such protection is yet to be established. It is presumed that once a person has been infected, his or her chance of getting a second infection 2-3 months later is low, but how long that protective immunity might last is not known [50].

The determination of the IgM antibody titer can confirm the current infection. Unlike molecular methods, which study the direct presence of SARS-CoV-2 in the body, serological methods indicate its presence indirectly [30]. These tests can facilitate the diagnosis of a SARS-CoV-2 infection when an NP swab specimen was collected inappropriately and the molecular assays were performed unsatisfactorily. Also, despite the ongoing infection, virus particles may be undetected, because the sensitivity of PCR tests decreases with time. Diagnostic laboratories perform tests in immunochemical analyzers using the ELISA method. Less sensitive and less specific rapid tests utilize the immunochromatographic method [36]. It is worth to articulate that serological tests are essential in epidemiological studies to understand the extent of the infection after it has ended. Until vaccine and proper antiviral treatment protocols are established, serological tests should not be underestimated [29].

\section{The ELISA method}

The ELISA method is a semi-quantitative method utilizing automated immunochemical analyzers in the detection of $\operatorname{IgM}, \operatorname{IgA}$ and IgG antibodies [29]. When discussing serological tests, the phenomenon of seroconversion should be mentioned. It is the qualitative and quantitative change in the serum antibodies during the infection. Due to the fact that SARS-CoV-2 is a new pathogen, it is not yet exactly established when the production of antibodies against the virus occurs. In the first stage of the immune response, IgM antibodies appear, which over time are replaced by more stable IgG antibodies. This relationship is used in the diagnosis of various infectious diseases. High levels of IgM and $\operatorname{IgG}$ usually indicate an active infection, while high levels of IgG and low levels of IgM indicate a past infection [30]. The IgM and IgG specific antibodies should be detectable on the fourth-fifth day of the infection $[23,24]$.
According to one study, IgM antibodies were detected in $70 \%$ of the patients with infection signs on the 8th-14th day of the infection, and in $90 \%$ of the patients on the 11th-24th day of the infection. The IgG antibodies to SARS-CoV-2 generally become detectable 10-14 days after the infection, although they may be detected earlier, and they normally peak around the 28th day after the infection onset. [26]. Another study showed that the presence of antibodies was $<40 \%$ among patients during the first 7 days of the disease, and then it rapidly increased to $94.3 \%$ and $79.8 \%$ for IgM and IgG, respectively, on the 15th day after the onset of the disease symptoms [45]. Similarly, in a study conducted by Guo et al. [55], analyzing 208 plasma samples, IgM, IgA and IgG were present in $85.4 \%, 92.7 \%$ and $77.9 \%$ of the specimens, respectively. On the other hand, in the group of 185 control samples (healthy subjects and patients with acute lower respiratory tract infections), no anti-SARS-CoV-2 IgM, IgA and IgG were detected. These authors also found strong cross-reactivity of the SARS-CoV serological test. In contrast, no cross-reactivity with other human coronaviruses (NL63, 229E, OC43, HKU1) was found.

Cross-reactivity was found in the research by Howard et al. [25]. According to some authors, at the current stage of development of serological tests, cross-reactivity with other coronaviruses can be a major challenge [37]. It is also puzzling whether such cross-reaction could give false-positive results in patients with autoimmune diseases.

\section{Rapid diagnostic tests based on antibody detection}

Among serological tests, qualitative rapid tests can also be distinguished. Most of these assays assess the presence of IgM and IgG simultaneously, based on the immunochromatographic methods which utilize gold particles. These tests are recommended by manufacturers as time- and cost-efficient ready-to-use screening tools for dental office use. They resemble pregnancy tests. With just a few drops of capillary blood obtained from a finger, they provide easy-to-interpret results, without the necessity for any diagnostic laboratory involvement [53]. These tests take $<20$ min [26]. However, they demonstrate very low sensitivity and specificity. They frequently give false-negative results, even in the second week of the infection, because of the lack of seroconversion. What is more, the antigen utilized in the immunochromatographic test is SARSr-111-CoV Rp3 nucleocapsid protein, which constitutes a high risk of 
cross-reactions with other coronaviruses and false-positive results [23]. Rapid tests used in the dental offices should only detect IgM-class antibodies that indicate an active COVID-19 infection. If the test assesses both IgM and IgG, a positive result may indicate a history of an inactive infection. It is also important to check if the test is approved for use in the European Union (a CE certificate), and if it could be used for testing human samples (an IVD certificate) [36].

Tests used to detect antibody responses to COVID-19 in the population will be critical in supporting the development of vaccines, and in adding to the general understanding of the extent of infections among people who are not identified through active case finding and surveillance efforts, the attack rate in the population, and the infection fatality rate. For clinical diagnosis, however, such tests have limited utility, because they cannot quickly diagnose acute infections and cannot provide the basis for informed actions needed to determine the course of treatment. While WHO does not recommend the use of antibody-detecting rapid diagnostic tests for outpatient care, it encourages the continuation of work to establish their usefulness in the disease surveillance and epidemiologic research [9].

\section{CONCLUSIONS}

Due to the fact that rapid serological tests are an unreliable source of medical data, they should not be utilized as a widespread screening tool in outpatient medical setting. False-negative results pose a huge danger of the spread of COVID-19 to medical personnel and other patients. False-positive results, on the other hand, may cause patients' anxiety. The most reliable methods of SARS-CoV-2 diagnostics are molecular tests such as RT-PCR, but because of the shortages in the diagnostic capacity during the COVID-19 pandemic and the financial aspect of the widespread laboratory diagnostics, it is impossible to provide them. Serological ELISA assays identify people who were infected with SARS-CoV-2 and have already recovered from COVID-19, but they do not give sufficient information about the acute infection. This is why the only means to mitigate the spread of COVID-19 in the outpatient clinical setting, such as a dental office, includes a thorough epidemiological interview, temperature measurement in order to rule out patients with active infections, and the implementation of strict infection control procedures.

\section{REFERENCES}

1. Chen N, Zhou M, Dong X, Qu J, Gong F, Han Y, et al. Epidemiological and clinical characteristics of 99 cases of 2019 novel coronavirus pneumonia in Wuhan, China: a descriptive study. Lancet. 2020;395(10223):507-13, https://doi.org/10.1016/S0140-6736(20)30211-7.

2. Wu JT, Leung K, Bushman M, Kishore N, Niehus R, Salazar De, et al. Estimating clinical severity of COVID-19 from the transmission dynamics in Wuhan, China. Nat Med. 2020;26 https://doi.org/10.1038/s41591-020-0822-7.

3. Coronaviridae Study Group of the International Committee on Taxonomy of Viruses. The species Severe acute respiratory syndrome-related coronavirus: classifying 2019-nCoV and naming it SARS-CoV-2. Nat Microbiol. 20202;5:53644, https://doi.org/10.1038/s41564-020-0695-z.

4. Zhu J, Ji P, Pang J, Zhong Z, Li H, He C. Clinical characteristics of 3,062 COVID-19 patients: a meta-analysis. J Med Virusol. 2020;92:1902-14, https://doi.org/10.1002/ jmv.25884.

5. The American Dental Association [Internet]. Chicago: The Association; 2020 [cited 2020 Jun 1]. ADA recommending dentists postpone elective procedures. Available from: https://www.ada.org/en/publications/ada-news/2020archive/march/ada-recommending-dentists-postponeelective-procedures.

6. Odeh ND, Babkair H, Abu-hammad S, Borzangy S. COVID-19: Present and Future Challenges for Dental Practice. J Environ Res Public. 2020;19(9):3151, https:// doi.org/10.3390/ijerph17093151.

7. American Dental Association [Internet]. Chicago: The Association; 2020 [cited 2020 Jun 1]. Available from: http://www.success.ada.org/en/practice-management/ patients/infectious-diseases-2019-novel-coronavirus.

8. World Health Organization [Internet]. Geneva: The Organization; 2020 [cited 2020 June 1]. WHO Coronavirus Disease (COVID-19) Dashboard. Available from: https:// covid19.who.int/?gclid=Cj0KCQjww_f2BRC-ARIsAP3zarEr-xP7RzyubT81rbZ98qekaQ49Szecu08bl4VxedF3-cPxDQAFnH0aAnQZEALw_wcB.

9. World Health Organization [Internet]. Geneva: The Organization; 2020 [cited 2020 June 1]. Advice on the use of point-of-care immunodiagnostic tests for COVID-19. Available from: https://www.who.int/news-room/commentaries/detail/advice-on-the-use-of-point-of-care-immunodiagnostic-tests-for-covid-19.

10. Gennaro FD, Pizzol D, Marotta C, Antunes M, Racalbuto V, Veronese N, et al. Coronavirus Diseases (COVID-19) Current Status and Future Perspectives: A Narrative Review. Int J Environ Res Public Health. 2020;17(8):2690, https://doi.org/10.3390/ijerph17082690. 
11. Chen Y, Liu Q, Guo D. Emerging coronaviruses: Genome structure, replication, and pathogenesis. J Med Virol. 2020;92(4):418-23, https://doi.org/10.1002/jmv.25681.

12. Domańska-Blicharz K, Kuczkowski M, Sajewicz-Krukowska J. Whole genome characterisation of quail deltacoronavirus detected in Poland. J Virus Genes. 2019;55(2):2437, https://doi.org/10.1007/s11262-019-01639-1.

13. Wang Q, Zhang Y, Wu L, Niu S, Song L, Zhang Z, et al. Structural and Functional Basis of SARS-CoV-2 Entry by Using Human ACE2. Cell. 2020;181(4):894-904, https:// doi.org/10.1016/j.cell.2020.03.045.

14. Peng X, Xu X, Li Y, Cheng L, Zhou X, Ren B. Transmission routes of 2019-nCoV and controls in dental practice. Int J Oral Sci. 2020;12(9), https://doi.org/10.1038/s41368-0200075-9.

15. Zhou P, Yang X, Wang X, Hu B, Zhang L, Zhang W, et al. A pneumonia outbreak associated with a new coronavirus of probable bat origin. Nature. 2020;579:270-3, https:// doi.org/10.1038/s41586-020-2012-7.

16. Wahba L, Jain N, Fire A, Shoura MJ, Artiles KL, McCoy MJ, et al. Identification of a pangolin niche for a 2019-nCoV-like coronavirus through an extensive meta-metagenomic search. BioRxiv. 2020, https://doi.org/ 10.1101/2020.02.08.939660.

17. Wu A, Peng Y, Huang B, Ding X, Wang X, Niu P, et al. Genome Composition and Divergence of the Novel Coronavirus (2019-nCoV) Originating in China. Cell Host Microbe. 2020;27(3):325-8, https://doi.org/10.1016/ j.chom.2020.02.001.

18. Lu R, Zhao X, Li J, Niu P, Yang B, Wu H, et al. Genomic characterisation and epidemiology of 2019 novel coronavirus : implications for virus origins and receptor binding. Lancet. 2020;395(10224):565-74, https://doi.org/10.1016/ S0140-6736(20)30251-8.

19. Zheng M. Novel antibody epitopes dominate the antigenicity of spike glycoprotein in SARS-CoV-2 compared to SARS-CoV. Cell Mol Immunol. 2020;17(5):536-8, https:// doi.org/10.1038/s41423-020-0385-z.

20. Rowan JN, Laffey JG. Challenges and solutions for addressing critical shortage of supply chain for personal and protective equipment (PPE) arising from Coronavirus disease (COVID19) pandemic - Case study from the Republic of Ireland. J Sci Total Environ. 2020;725:138532, https://doi. org/10.1016/j.scitotenv.2020.138532.

21. Gabutti G, Federica A. Coronavirus: Update Related to the Current Outbreak of COVID-19. J Infect Dis Ther. 2020;9(2):241-53, https://doi.org/10.1007/s40121-02000295-5.

22. Ather A, Patel B, Ruparel NB, Diogenes A, Hargreaves KM. Coronavirus Disease 19 (COVID-19): Implications for
Clinical Dental Care. J Endod. 2020;46(5):584-95, https:// doi.org/10.1016/j.joen.2020.03.008.

23. Loeffelholz MJ, Tang Y. Laboratory diagnosis of emerging human coronavirus infections - the state of the art. Emerg Microbes Infect. 2020;9:747-56, https://doi.org/10.1080/ 22221751.2020 .1745095$.

24. Coulthard P. Dentistry and coronavirus (COVID-19) moral decision-making. Br Dent J. 2020;228(7):503-5, https://doi.org/10.1038/s41415-020-1482-1.

25. Wan W, Lim S, Seng E. Cross-reaction of sera from COVID-19 patients with SARS-CoV assays. MedRxiv. 2020, https://doi.org/10.1101/2020.03.17.20034454.

26. Beeching NJ, Fletcher TE, Beadsworth MBJ. Covid-19: testing times Rapid near patient testing for both current and past infections is urgently required. BMJ. 2020;369:m1403, https://doi.org/doi:10.1136/bmj.m1403.

27. Mizumoto K, Kagaya K, Zarebski A, Chowell G. Estimating the asymptomatic proportion of coronavirus disease 2019 (COVID-19) cases on board the Diamond Princess cruise ship, Yokohama, Japan, 2020. J Euro Surveill. 2020;25(10):2000180, https://doi.org/10.2807/1560-7917. ES.2020.25.10.2000180

28. Wenling W, Xu Y, Gao R, Al. E. Detection of SARS-CoV-2 in Different Types of Clinical Specimens. JAMA. 2020; 323(18):1843-4, https://doi.org/10.1001/jama.2020.3786.

29. Gp JW, Penny F. Interpreting a covid-19 test result. BMJ. 2020;369:m1808, https://doi.org/10.1136/bmj.m1808.

30. Karabin K [Internet]. Medycyna Praktyczna; 2020 [cited 2020 Jun 1]. Diagnostyka laboratoryjna COVID-19. Available from: https://www.mp.pl/pacjent/badania_zabiegi/234787, diagnostyka-laboratoryjna-covid-19.

31. Sabino-Silva R, Carolina A, Jardim G, Siqueira WL. Coronavirus COVID-19 impacts to dentistry and potential salivary diagnosis Coronavirus COVID-19 impacts to dentistry and potential salivary diagnosis. Clin Oral Investig. 2020;24(4):1619-21, https://doi.org/10.1007/s00784-02003248-x.

32. Na Z, Dingyu Z, Wenling W, Xingwang L, Bo Y, Jingdong S, et al. A Novel Coronavirus from Patients with Pneumonia in China, 2019. N Engl J Med. 2020;382(8):727-33, https:// doi.org/10.1056/NEJMoa2001017.

33. Santosh TS, Parmar R, Anand H, Srikanth K. A Review of Salivary Diagnostics and Its Potential Implication in Detection of Covid-19. Cureus. 2020;12(4):e7708, https:// doi.org/10.7759/cureus.7708.

34. Wyllie AL, Fournier J, Casanovas-Massana A, Campbell M, Tokuyama M, Vijayakumar P, et al. Saliva is more sensitive for SARS-CoV-2 detection in COVID-19 patients than nasopharyngeal swabs. MedRxiv. 2020, https://doi. org/10.1101/2020.04.16.20067835. 
35. U.S. Food and Drug Administration [Internet]. Silver Spring: The Administration; 2020 [cited 2020 Jun 1]. Coronavirus (COVID-19) Update: FDA Authorizes First Diagnostic Test Using At-Home Collection of Saliva Specimens. Available from: https://www.fda.gov/news-events/press-anno uncements/coronavirus-covid-19-update-fda-authorizes-first-diagnostic-test-using-home-collection-saliva.

36. Bielawska-Drózd A, Michalski A, Cieślik P, Makowski P, Kocik J. Metody diagnostyki laboratoryjnej COVID-19. Wiedza Med. 2020;1-9, https://doi.org/10.36553/wm.41.

37. World Health Organization [Internet]. The Organization, Geneva 2020 [cited 1st June 2020]. "Immunity passports" in the context of COVID-19. Available from: https://www. who.int/news-room/commentaries/detail/immunitypassports-in-the-context-of-covid-19.

38. Xiao AT, Gao C, Zhang S. Profile of specific antibodies to SARS-CoV-2: The first report. J Infect. 2020;S01634453(20)30138-9,https://doi.org/10.1016/j.jinf.2020.03.012.

39. Jääskeläinen AJ, Kekäläinen E, Kallio-Kokko H, Mannonen L, Kortela E, Vapalahti O, et al. Evaluation of commercial and automated SARS-CoV-2 IgG and IgA ELISAs using coronavirus disease (COVID-19) patient samples. Euro Surveill. 2020;25(18):2000603, https://doi.org/ 10.2807/1560-7917.ES.2020.25.18.2000603.

40. Huang C, Wang Y, Li X, Ren L, Zhao J, Hu Y, et al. Clinical features of patients infected with 2019 novel coronavirus in Wuhan, China. Lancet. 2020;395(10223):497-506, https://doi.org/10.1016/S0140-6736(20)30183-5.

41. Song F, Lu H. Emerging 2019 Novel Coronavirus (2019-nCoV) Pneumonia. Radiology. 2020;295(8):210-7, https://doi.org/10.1148/radiol.2020200274.

42. Peng Q-Y, Wang X-T, Zhang L-N. Findings of Lung Ultrasonography of Novel Corona Virus Pneumonia During the 2019-2020 Epidemic. Intensive Care Med. 2020;46(5): 849-50, https://doi.org/10.1007/s00134-020-05996-6.

43. Huang G, Kovalic AJ, Graber CJ. Prognostic Value of Leukocytosis and Lymphopenia for Coronavirus Disease Severity. Emerg Infect Dis. 2020;26(8), https://doi.org/ 10.3201/eid2608.201160.

44. Yan Y, Chang L, Wang L. Laboratory testing of SARS-CoV, MERS-CoV, and SARS-CoV-2 (2019-nCoV): Current status, challenges, and countermeasures. Rev Med Virusol. 2020;30(3):e2106, https://doi.org/10.1002/rmv.2106.

45. Juanjuan Z, Quan Y, Haiyan W, Wei L, Xuejiao L, Yingying S, et al. Antibody responses to SARS-CoV-2 in patients of novel coronavirus disease 2019. Clin Infect Dis. 2020;ciaa344, https://doi.org/10.1093/cid/ciaa344.

46. Broughton JP, Deng X, Yu G, Fasching CL, Singh J, Streithorst J, et al. Rapid Detection of 2019 Novel Coronavirus SARS-CoV-2 Using a CRISPR-based DETECTR Lateral Flow Assay. MedRxiv. 2020, https://doi.org/10.1101/2020. 03.06.20032334.

47. Tang YW, Schmitz JE, Persing DH, Stratton CW. Laboratory Diagnosis of COVID-19: Current Issues and Challenges. J Clin Microbiol. 2020;158:e00512-20, https://doi. org/10.1128/JCM.00512-20.

48. Xiao AT, Tong YX, Zhang S. Profile of RT-PCR for SARSCoV-2: a preliminary study from 56 COVID-19 patients. Clin Infect Dis. 2020, https://doi.org/10.1093/cid/ciaa460.

49. Yu L, Wu S, Hao X, Dong X, Mao L, Pelechano V, et al. Rapid Detection of COVID-19 Coronavirus Using a Reverse Transcriptional Loop-Mediated Isothermal Amplification (RT-LAMP) Diagnostic Platform. Clin Chem. 2020, https://doi.org/10.1093/clinchem/hvaa102.

50. Kontou PI, Braliou GG, Dimou NL, Nikolopoulos G, Bagos PG. Antibody tests in detecting SARS-CoV-2 infection: a meta-analysis. MedRxiv. 2020, https://doi.org/ 10.1101/2020.04.22.20074914.

51. Park G, Ku K, Baek S, Kim S, Kim S Il, Kim B, et al. Development of Reverse Transcription Loop-Mediated Isothermal Amplification Assays Targeting SARS-CoV-2. J Mol Diagnostics. 2020;22(6):729-35, https://doi.org/10. 1016/j.jmoldx.2020.03.006.

52. West C, Montori W, Sampathkumar P. COVID-19 Testing. The Threat of False-Negative Results. Mayo Clin Proc. 2020;95(6):1127-9, https://doi.org/10.1016/j.mayocp. 2020.04.004.

53. Kubina R, Dziedzic A. Molecular and Serological Tests for COVID-19. A Comparative Review of SARS-CoV-2 Coronavirus Laboratory and Point-of-Care Diagnostics. Diagnostics. 2020;10(434), https://doi.org/10.3390/diagnostics 10060434 .

54. Liu L, Liu W, Wang S, Zheng S. A preliminary study on serological assay for severe acute respiratory syndrome coronavirus 2 (SARS-CoV-2) in 238 admitted hospital patients. MedRxiv. 2020, https://doi.org/10.1101/2020.03. 06.20031856 .

55. Guo L, Ren L, Yang S, Xiao M, Chang D, Yang F, et al. Profiling Early Humoral Response to Diagnose Novel Coronavirus Disease (COVID-19). Clin Infect Dis. 2020; ciaa310, https://doi.org/10.1093/cid/ciaa310.

This work is available in Open Access model and licensed under a Creative Commons Attribution-NonCommercial 3.0 Poland License - http://creativecommons.org/licenses/by-nc/3.0/pl/deed.en. 